\title{
SURFACE AREA OF A CONVEX BODY UNDER AFFINE TRANSFORMATIONS
}

\author{
C. M. PETTY
}

1. Introduction. Let $K$ be a nondegenerate convex body in $E^{n}$. We will seek requirements on $K$ such that its surface area is minimal compared to that of its affine transforms of the same volume. This problem has been studied for $n=2$ by Behrend [1], Green [2], and Gustin [3].

2. Necessary and sufficient conditions. Let $S$ denote the surface of $K, V(K)$ its volume and $S(K)$ its surface area.

THEOREM 1. Necessary and sufficient conditions that a nondegenerate convex body $K$ in $E^{n}$ have minimal surface area among its affine transforms of volume $V(K)$ are that

$$
\int_{s} u_{i} u_{j} d S_{u}=0
$$

for $i \neq j$

and

$$
\int_{S} u_{i}^{2} d S_{u}=\frac{1}{n} S(K)
$$

for $i, j=1, \cdots, n$ where $\left(u_{1}, \cdots, u_{n}\right)$ is the outer unit vector normal to $S$ at the point with surface area element $d S_{u}$.

PROOF. We need only consider central affine transformations of the type $(x, y$ are column vectors of length $n)$

$$
y=B x, \quad \operatorname{det}(B)=1 .
$$

Suppose that the convex body $K_{i}(i=1, \cdots, n)$ is transformed into $K_{i}^{*}$ by (1), then the linear combination $\lambda_{1} K_{1}+\cdots+\lambda_{n} K_{n}$ is transformed into $\lambda_{1} K_{1}^{*}+\cdots+\lambda_{n} K_{n}^{*}$. Since volume is preserved, it follows from the definition of mixed volumes $[4$, p. 38] that

$$
V\left(K_{p_{1}}, \cdots, K_{p_{n}}\right)=V\left(K_{p_{1}}^{*}, \cdots, K_{p_{n}}^{*}\right) .
$$

Let $E$ be an ellipsoid with supporting function $E(u)$, then

$$
n V(K, \cdots, K, E)=\int_{\mathcal{s}} E(u) d S_{u}, \quad|u|=1 .
$$

Received by the editors September 15, 1960. 
It is clear, then, that our problem is equivalent to keeping $K$ fixed and minimizing $V(K, \cdots, K, E)$ over all ellipsoids with center at the origin and volume

$$
\kappa_{n}=\frac{\pi^{n / 2}}{\Gamma(n / 2+1)} .
$$

To show the existence of a minimizing ellipsoid, let $U$ be a solid sphere, of radius $r>0$, contained in $K$ and let $\bar{a}$, of length $a$, be a segment of maximum length contained in $E$. By the inclusion property of mixed volumes $[4$, p. $41,(5)]$ and the representation $[4$, p. 45$]$ for the area of the projection of $U$, we have $V(K, \cdots, K, E)$ $\geqq V(U, \cdots, U, \bar{a})=a r^{n-1} n^{-1} \kappa_{n-1}$. Consequently, a sequence of ellipsoids $E_{i}$ of the above type, for which $V\left(K, \cdots, K, E_{i}\right)$ tends to the g.l.b. of (3), is uniformly bounded and by Blaschke's selection theorem $\left[4\right.$, p. 34] there exists a subsequence of the $E_{i}$ which converges to a convex body $E^{*}$. The body $E^{*}$ is necessarily an ellipsoid and, by the continuity of mixed volumes, $E^{*}$ is a minimizing ellipsoid.

Now, let $A^{-1}$ be a positive definite symmetric matrix with $\operatorname{det}\left(A^{-1}\right)$ $=1$. If $E$ is the ellipsoid with surface

$$
x^{T} A^{-1} x=1
$$

(the superscript $T$ meaning transpose) then the polar reciprocal of $E$ with respect to the origin has distance function

$$
E(u)=\left\{u^{T} A u\right\}^{1 / 2} .
$$

The above is clear if $A^{-1}$ is a diagonal matrix and the general case follows by considering an orthogonal transformation. Consequently, (6) is the supporting function of $E$, see [4, p. 28], and if $E$ is transformed into $E^{*}$ by (1) then $E^{*}(u)$ is found by replacing $A$ in (6) by $B A B^{T}$.

Let $F(B)$ be the function of $n^{2}$ variables $b_{i j}$ given by

$$
F(B)=\int_{S} E_{\Delta}(u) d S_{u}, \quad|u|=1,
$$

where $E_{A}(u)$ is given by (6) and $A=B B^{T}$. Since $B B^{T}$ is positive semidefinite, (7) is defined for all $B$. We wish to minimize (7), with the constraint

$$
\operatorname{det}(B)=1 \text {. }
$$

If we equate to zero the partial derivatives of $F(B)+\lambda(1-\operatorname{det}(B))$ and use (8) we have 


$$
\left[\frac{\partial F}{\partial b_{i j}}\right]=\lambda\left(B^{T}\right)^{-1}
$$

Also

$$
\left[\frac{\partial F}{\partial b_{i j}}\right]=\left[\int_{S} u_{i} u_{j} E_{A}^{-1}(u) d S_{u}\right] B
$$

Consequently,

$$
A^{-1}=\frac{1}{\lambda}\left[\int_{S} u_{i} u_{j} E_{A}^{-1}(u) d S_{u}\right], \quad \lambda=\frac{1}{n} \int_{S} E_{A}(u) d S_{u} .
$$

If $A$ is any positive definite symmetric matrix with $\operatorname{det}(A)=1$ which satisfies (11) then the ellipsoid with supporting function (6) will be called a critical ellipsoid of $K$. It is clear that the unit matrix satisfies (11) if and only if the conditions in Theorem 1 are satisfied. Consequently, a proof of the following result completes the proof of Theorem 1.

THEOREM 2. If $K$ is a nondegenerate convex body with surface $S$, then there exists one and only one positive definite symmetric matrix $A$ with $\operatorname{det}(A)=1$ such that

$$
A^{-1}=\frac{n}{\int_{S} E_{A}(u) d S_{u}}\left[\int_{S} u_{i} u_{j} E_{A}^{-1}(u) d S_{u}\right]
$$

where $E_{A}(u)$ is given by (6).

Proof. Suppose $C$ is the matrix of an affine transformation of type (1) which carries $K$ into $K^{*}$. If $F^{*}(D)$ is the corresponding function (7) for $K^{*}$ then

$$
F(B)=F^{*}(C B)
$$

for all $B$. By (2) and (3), this is the case for $B$ with $\operatorname{det}(B)=1$ and by the special form of (7) this may be extended to all $B$. We have then

$$
\left[\frac{\partial F}{\partial b_{i j}}\right]=C^{T}\left[\frac{\partial F^{*}}{\partial d_{i j}}\right], \quad D=C B .
$$

Consequently, by (11), (13), (10) and (12) we conclude that a critical ellipsoid of $K$ is carried by $C$ into a critical ellipsoid of $K^{*}$. We may, therefore, assume that the solid unit sphere is a critical ellipsoid of $K$ and consequently that the conditions in Theorem 1 hold. Let 


$$
E(a, u)=\left[\sum_{1}^{n} a_{i}^{2} u_{i}^{2}\right]^{1 / 2}
$$

and

$$
F(a)=\frac{1}{S(K)} \int_{S} E(a, u) d S_{u} .
$$

The function $F(a)$ is defined for all points $a$ in $E^{n}$ and has the following properties:

(1) $F(a)>0$ for $a \neq 0$ and $F(0)=0$,

(2) $F(\lambda a)=\lambda F(a), \lambda>0$,

(3) $F(a+b) \leqq F(a)+F(b)$.

To prove property (3), we have by Cauchy's inequality

$$
\sum a_{i} b_{i} u_{i}^{2} \leqq\left[\left(\sum a_{i}^{2} u_{i}^{2}\right)\left(\sum b_{i}^{2} u_{i}^{2}\right)\right]^{1 / 2}
$$

and consequently

$$
E(a+b, u) \leqq E(a, u)+E(b, u) .
$$

By [4, pp. 21-22], $F(a)$ is the distance function of a convex body and, by the conditions in Theorem 1 , this convex body lies entirely in the closed half-space containing the origin and bounded by the tangent plane $\sum_{1}^{n} x_{i} / n^{1 / 2}=n^{1 / 2}$. Now let $M$ be the set of all points $a$ such that $a_{i}>0$ and $a_{1} \cdots a_{n}=1$. By the inequality between the geometric and arithmetic means we have $\sum_{1}^{n} a_{i} / n^{1 / 2} \geqq n^{1 / 2}$ for all points in $M$ with equality only if $a_{i}=1(i=1, \cdots, n)$. Consequently, $F(a)$ evaluated over all points in $M$ has a unique absolute minimum for $a=(1, \cdots, 1)$. This completes the proof of Theorem 2 since the conditions in Theorem 1 are invariant under an orthogonal transformation.

3. The projection body. Green [2] has shown that for $n=2$ the conditions in Theorem 1 may be replaced by the requirement that the second Fourier coefficients of the supporting function $H(\cos \theta, \sin \theta)$ $=h(\theta)$ of $K$ vanish. We may generalize this to $n$-dimensions if in place of the supporting function of $K$ we use the supporting function $\sigma(u)$ of the projection body of $K$, see [4, p. 45]. This may be viewed as a generalization since for $n=2, \sigma(\cos \theta, \sin \theta)=h(\theta+\pi / 2)+h(\theta-\pi / 2)$.

TheOREM 3. If $K$ is a nondegenerate convex body whose projection body has supporting function $\sigma(u)$, then necessary and sufficient conditions that the surface area of $K$ be minimal among its affine transforms of volume $V(K)$ are that 


$$
\int_{\Omega} u_{i} u_{j} \sigma(u) d \omega_{u}=0 \quad \text { for } i \neq j
$$

and

$$
\int_{\Omega}^{2} u_{i}^{2} \sigma(u) d \omega_{u}=\frac{\kappa_{n-1}}{n} S(K), \quad|u|=1
$$

for $i, j=1, \cdots, n$ where the integration is extended over the surface $\Omega$ of the solid unit sphere. by

Proof. The supporting function $\sigma(u)$ is given, see [4, pp. 48-49],

$$
\sigma(u)=\frac{1}{2} \int_{S}|u \cdot \tau| d S_{\tau}, \quad|\tau|=1
$$

and

$$
S(K)=\frac{1}{\kappa_{n-1}} \int_{\Omega} \sigma(u) d \omega_{u}, \quad|u|=1 .
$$

Let $S_{2}(u)$ be any surface harmonic of degree 2 , then by the FunkHecke theorem [5, pp. 247-248] we have for unit vectors $u$ and $\tau$

$$
\int_{\Omega}|u \cdot \tau| S_{2}(u) d \omega_{u}=\frac{\pi^{(n-1) / 2}}{\Gamma((n+3) / 2)} S_{2}(\tau) .
$$

Consequently, by (16),

$$
\int_{\Omega} S_{2}(u) \sigma(u) d \omega_{u}=\frac{1}{2} \frac{\pi^{(n-1) / 2}}{\Gamma((n+3) / 2)} \int_{S} S_{2}(\tau) d S_{\tau}
$$

and Theorem 3 follows directly from Theorem 1 and (17).

\section{REFERENCES}

1. F. Behrend, Über einige Affininvariaten konvexer Bereiche, Math. Ann. vol. 113 (1937) pp. 713-747.

2. J. W. Green, Length and area of a convex curve under affine transformation, Pacific J. Math. vol. 3 (1953) pp. 393-402. 405.

3. W. Gustin, $A n$ isoperimetric minimax, Pacific J. Math. vol. 3 (1953) pp. 403-

4. T. Bonnesen and W. Fenchel, Theorie der konvexen Körper, New York, Chelsea Publishing Co., 1948.

5. A. Erdelyi et al., Higher transcendental functions, Bateman Manuscript Project, vol. 2, New York, McGraw-Hill, 1953, pp. 247-248.

Lockreed Missiles and Space Division 